\title{
Conservative Hepatectomy for Tumors Involving the Middle Hepatic Vein and Segment 1: The Liver Tunnel
}

\author{
Guido Torzilli, MD, PhD, FACS, Matteo Cimino, MD, Fabio Procopio, MD, Guido Costa, MD, Matteo Donadon, \\ MD, Daniele Del Fabbro, MD, Andrea Gatti, MD, and Carlos A. Garcia-Etienne, MD
}

Department of Hepatobiliary Surgery, Humanitas Research Hospital, University of Milan-School of Medicine, Rozzano, Milan, Italy

\begin{abstract}
Background. For lesions invading the middle hepatic vein (MHV) at caval confluence (CC) the mini-mesohepatectomy(MMH) was proposed. ${ }^{1}$ If the lesion is extended to the paracaval portion of segment $1(\mathrm{~S} 1)$ in contact or invading the MHV a new procedure is proposed.

Methods. Case-1: mass forming cholangiocarcinoma (MFCCC) $4 \mathrm{~cm}$ in size invading the MHV and in contact with right (RHV) and left hepatic vein (LHV) at the CC. In Case-2, two colorectal liver metastases (CLM) both $2 \mathrm{~cm}$ in size occupied S1 (T1) and S8 (T2): T1 was located between RHV and the inferior vena cava (IVC), T2 was in contact with MHV at CC. According to tumor-vessel intraoperative-ultrasound classification ${ }^{2}$ and color-flow analysis ${ }^{3}$ parenchyma-sparing procedure was performed.

Results. In Case-1 a communicating vein (CV) between RHV and MHV was detected at color-flow-IOUS. Contacts between MFCCC with RHV and LHV were confirmed at IOUS as detachable. In Case- 2 contact between T1 with MHV was confirmed at IOUS as detachable. Liver-tunnel with IVC and main portal vein bifurcation exposure was performed resecting the MHV in Case- 1 and preserving it
\end{abstract}

This video was accepted and presented at the Hepatobiliary Surgery video session of the 99th Annual Clinical Congress of the American College of Surgeons.

Electronic supplementary material The online version of this article (doi:10.1245/s10434-014-3675-9) contains supplementary material, which is available to authorized users.

(C) Society of Surgical Oncology 2014

First Received: 6 January 2014;

Published Online: 11 April 2014

G. Torzilli, MD, PhD, FACS

e-mail: guido.torzilli@humanitas.it in Case-2. Both patients had ad an uneventful postoperative course and were discharged on the 8th postoperative day. Conclusion. For tumors involving S1, S4s and/or S8 and infiltrating or in contact with the MHV at the $\mathrm{CC}$, can be removed in a conservative manner by means of the herein described "Liver Tunnel" approach. The latter introduces a further step in favour of parenchyma-sparing policy for centrally located lesions with complex tumor-vessel relationship.

\section{REFERENCES}

1. Torzilli G, Botea F, Donadon M, et al. Minimesohepatectomy for colorectal liver metastasis invading the middle hepatic vein at the hepatocaval confluence. Ann Surg Oncol. 2010;17:483.

2. Torzilli G, Montorsi M, Del Fabbro D, et al. Ultrasonographically guided surgical approach to liver tumours involving the hepatic veins close to the caval confluence. Br J Surg. 2006;93:1238-46.

3. Torzilli G, Garancini $M$, Donadon $M$, et al. Intraoperative ultrasonographic detection of communicating veins between adjacent hepatic veins during hepatectomy for tumours at the hepatocaval confluence. Br J Surg. 2010;97:1867-73. 